\title{
The Passive Location of Emitter based on Fuzzy Method
}

\author{
Wanli Xu, Zhun Liu, Huiyu Nie \\ Department of Information Engineering \\ Academy of Armored Force Engineering \\ Beijing,100072,China \\ E-mail: xuwanli631101@sina.com
}

\begin{abstract}
The Passive Location of Emitter based on Fuzzy Method.[Purpose] The use of passive observation station to achieve the precise location has been an important topic of monitoring the electromagnetic spectrum resources and the environment. In order to locate the emitter accurately, a new method called fuzzy method is presented in this papers. [Method]Based on the distribution of points of intersections that are figured out by the $\mathrm{LOB}$ (Lines of Bearing), the paper put forward a fuzzy method for the passive location of emitter based on membership function to achieve the location of emitter. We compared this method with Pages method using 100 sets experimental data through the Monte Carlo simulated experiment. [Results] The results showed that this method can effectively achieve the location of emitter, and improve the location accuracy. [Conclusions] The positioning precision of this algorithm is obviously superior to Pages algorithm.
\end{abstract}

Keywords- degree of correlation; the fuzzy-location method; Pages algorithm

\section{INTRODUCTION}

With the rapid development of military electronic techniques, electronic warfare is more and more important in the modern war. It has gotten more and more attentions that how to improve the accuracy of emitter location effectually. We can obtain a set of direction measurements from the reconnaissance platforms by detecting the emitter. According to the direction measurements, we can estimate the location of emitter by dealing with the direction measurements.

There are a few location algorithms such as Probability Algorithm, Kalman Filter and Gradient Descending Algorithm and so on [1]. Least-Squared Triangle Location Algorithm and Pages-Zamora Algorithm are the two popular algorithms in the location of emitter. Especially PagesZamora Algorithm is applied in the cellular phone system by FCC (Federal Communication Commission) [2].

In this paper, according to the points of intersection calculated by LOB, we raise a fuzzy method for the passive location of emitter based on membership function[3] to achieve the location. At last, we compare the experimental results. The simulation experiment demonstrates that the results by using this Algorithm are better than the results by using Pages-Zamora Algorithm.

\section{II. PRINCIPLE OF LOCATION}

Assuming that the emitter is stationary and emits electromagnetic signals, the positions of reconnaissance platforms and emitter target are shown in Figure I. There are
$\mathrm{N}$ reconnaissance platforms that their coordinates are $\left(x_{i}, y_{i}\right)$ and their corresponding direction measures are $\varphi_{i}$, where $i=1,2, \cdots, N$. The coordinate of emitter target is $\left(x_{T}, y_{T}\right)$.

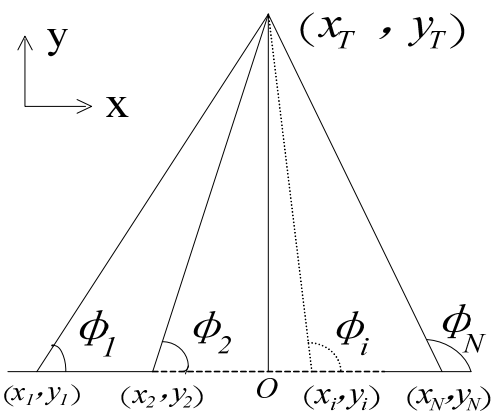

Figure 1. The positions of reconnaissance platforms and emitter target

The points of intersection calculated by direction measurements of reconnaissance platforms should be the same point in the ideal condition that there is no noise and measuring errors. However, the direction measurements always contain noise and measuring errors. As a result, the LOBs of reconnaissance platforms will not intersect at the same point. It is emphasis of location that how to deal with the data and estimate the target so that there is less effect in the estimators.

Assuming the variable to estimate determinate is the basic estimator problem[4]. When measuring the variable a few times, the measurements will not be same because of noise and measuring errors. So it needs an algorithm to estimate the variable according to the measurements legitimately. It is important to select a location algorithm.

\section{PAges-Zamora Algorithm}

Pages-Zamora Algorithm is a high-precision location method which is applied in the cellular phone system by the federal communications commission. Figure2 shows the principle of Pages-Zamora Algorithm.

The model of Pages-Zamora Algorithm is followed as below:

$$
r=r_{i}+d_{i} v_{i} \quad i=1,2, \cdots, N
$$




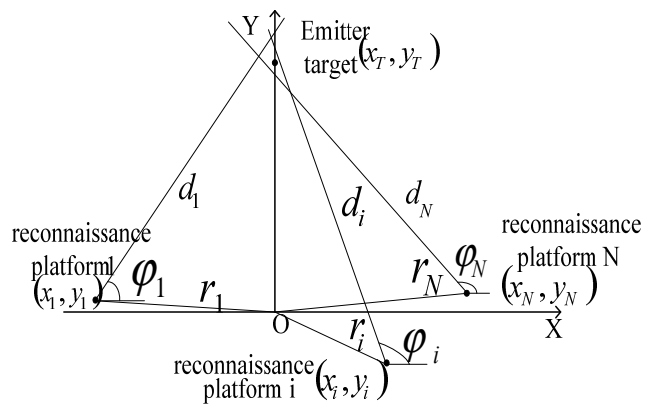

Figure 2. Principle of Pages-Zamora Algorithm

Where $d_{i}$ is the distance between the emitter target and i-th reconnaissance platform which its direction vector is $v_{i}$.

$$
v_{i}=\left[\cos \varphi_{i}, \sin \varphi_{i}\right]^{T}
$$

Considering equation (1),

$$
r=\left[\begin{array}{l}
x_{T} \\
y_{T}
\end{array}\right]=\left[\begin{array}{l}
x_{i} \\
y_{i}
\end{array}\right]+d_{i}\left[\begin{array}{c}
\cos \varphi_{i} \\
\sin \varphi_{i}
\end{array}\right]
$$

Dealing with the measurements of all reconnaissance platforms and eliminating $d_{i}$ in the equations, we can get the matrix form equations:

$$
\left[\begin{array}{c}
-x_{1} \sin \varphi_{1}+y_{1} \cos \varphi_{1} \\
-x_{2} \sin \varphi_{2}+y_{2} \cos \varphi_{2} \\
\vdots \\
-x_{N} \sin \varphi_{N}+y_{N} \cos \varphi_{N}
\end{array}\right]=\left[\begin{array}{cc}
-\sin \varphi_{1} & \cos \varphi_{1} \\
-\sin \varphi_{2} & \cos \varphi_{2} \\
\vdots & \vdots \\
-\sin \varphi_{N} & \cos \varphi_{N}
\end{array}\right]\left[\begin{array}{c}
x_{T} \\
y_{T}
\end{array}\right]
$$

we can write in this way:

$$
\begin{gathered}
a(\varphi)=H(\varphi) x \\
a(\varphi)=\left[\begin{array}{c}
-x_{1} \sin \varphi_{1}+y_{1} \cos \varphi_{1} \\
-x_{2} \sin \varphi_{2}+y_{2} \cos \varphi_{2} \\
\vdots \\
-x_{N} \sin \varphi_{N}+y_{N} \cos \varphi_{N}
\end{array}\right] \\
H(\varphi)=\left[\begin{array}{cc}
-\sin \varphi_{1} & \cos \varphi_{1} \\
-\sin \varphi_{2} & \cos \varphi_{2} \\
\vdots & \vdots \\
-\sin \varphi_{N} & \cos \varphi_{N}
\end{array}\right], \\
x=\left[\begin{array}{c}
x_{T} \\
y_{T}
\end{array}\right]
\end{gathered}
$$

Generally $N>2$, the number of equations is more than that of target's dimensions. So the least square solution of equation (3) is:

$$
\hat{x}=\left[H^{T}(\varphi) H(\varphi)\right]^{-1} H^{T}(\varphi) a(\varphi)
$$

\section{FUZZY ALGORITHM}

We can get a set of points of intersection calculated by direction measurements of reconnaissance platforms. According to any two reconnaissance platforms $\left(x_{i}, y_{i}\right)$ and $\left(x_{j}, y_{j}\right)$ where $i, j=1,2, \cdots, N$, we can get a point of intersection $\left(x_{i j}, y_{i j}\right)$ :

$$
\begin{aligned}
x_{i j} & =\frac{x_{j} \tan \varphi_{j}-x_{i} \tan \varphi_{i}+y_{i}-y_{j}}{\tan \varphi_{j}-\tan \varphi_{i}} \\
y_{i j} & =\frac{\left(x_{j}-x_{i}\right) \tan \varphi_{i} \tan \varphi_{j}+y_{i} \tan \varphi_{j}-y_{j} \tan \varphi_{i}}{\tan \varphi_{j}-\tan \varphi_{i}}
\end{aligned}
$$

So we can obtain $C_{N}^{2}$ points of intersection according to every two reconnaissance platforms. These points of intersection should concentrate around the target. The fuzzy method locate the target according to the degree of correlation of points each other[3]. Because the measure errors are caused by various kinds of factors, we can consider the error obey with Gause Distribution.

Assuming the points of intersection are $\left(x_{m}^{T}, y_{m}^{T}\right), m=1,2, \cdots, C_{N}^{2}$, we can calculate the degrees of correlation ${ }^{w_{m n}}$ of every point $\left(x_{n}^{T}, y_{n}^{T}\right), n=1,2, \cdots, C_{N}^{2}$ to the point $\left(x_{m}^{T}, y_{m}^{T}\right), m=1,2, \cdots, C_{N}^{2}$. We define the membership function is

$$
\begin{aligned}
& w_{m n}=\exp \left(\frac{\left(x_{n}^{T}-x_{m}^{T}\right)^{2}}{s_{x}^{2}}\right. \\
& \left.-\frac{2 r\left(x_{n}^{T}-x_{m}^{T}\right)\left(y_{n}^{T}-y_{m}^{T}\right)}{s_{x} s_{y}}+\frac{\left(y_{n}^{T}-y_{m}^{T}\right)^{2}}{s_{y}^{2}}\right)
\end{aligned}
$$

where $S_{x}$ is the standard deviation of $x$ coordinate of points, ${ }^{s}{ }$ is the standard deviation of ${ }^{y}$ coordinate of points and $r$ is the correlation coefficient of $x$ coordinate and ${ }^{y}$ coordinate of points. The degree of correlation of every point itself $w_{m n}$ is 1 . There are $C_{N}^{2}$ degrees of correlation $w_{m n}, m=1,2, \cdots, C_{N}^{2}$ to every point $\left(x_{n}^{T}, y_{n}^{T}\right), n=1,2, \cdots, C_{N}^{2}$. So we can get a $C_{N}^{2} \times C_{N}^{2}$ fuzzy matrix $F=\left(w_{m n}\right)_{C_{N}^{2} \times C_{N}^{2}}$ where $w_{m n}$ is the degree of correlation of point $\left(x_{n}^{T}, y_{n}^{T}\right)$ to point $\left(x_{m}^{T}, y_{m}^{T}\right)$. The n-th column of matrix $\mathrm{F}$ is the degrees of correlation of point 
$\left(x_{n}^{T}, y_{n}^{T}\right), n=1,2, \cdots, C_{N}^{2}$

to every point. Then we can calculate $a_{n}=\sum_{m=1}^{C_{N}^{2}} w_{m n}, n=1,2, \cdots, C_{N}^{2}$.where $a_{n}$ is the sum of degrees of correlation of point $\left(x_{n}^{T}, y_{n}^{T}\right)$ to every point. Make the point whose ${ }^{a_{n}}$ is max the last estimator.

Assuming that there are $\mathrm{N}$ reconnaissance platforms, it needs

$$
\frac{1}{8}\left(3 N^{6}-9 N^{5}+33 N^{4}-51 N^{3}+56 N^{2}-32 N\right)
$$

multiplication

operations and

$$
\frac{1}{8}\left(9 N^{6}-27 N^{5}+27 N^{4}-9 N^{3}+32 N^{2}-32 N\right)
$$

addition operations to run the fuzzy algorithm. When $\mathrm{N}$ is very large, we use (3/8)N6 multiplication operations to indicate the operations of fuzzy algorithm.

\section{SIMULATED EXPERIMENT}

In order to explain the principle, the emitter and reconnaissance platforms locate as FigureI. The emitter locates at $y$ coordinate axis and the reconnaissance platforms locate at $x$ coordinate axis. The reconnaissance platforms locate in same interval at every side of origin. Every reconnaissance platform generates random direction measurements by Monte Carlo[5] method. Make sure every reconnaissance platform work at the same time and get 100 sets of measurements to avoid contingency. At last, we compare the results through the mean and standard deviation.

Detecting the target a few times, we can get the location $\left(x_{T}, y_{T}\right)$ and its standard deviation $\left(s_{x_{T}}, s_{y_{T}}\right)$. The E.E.P (ellipse error probable) is calculated as below:

$$
\text { E.E.P }=\sqrt{s_{x_{T}}^{2}+s_{y_{T}}^{2}}
$$

Assuming the emitter target is stationary and its coordinate is $(0,100)$, distribute 11 reconnaissance platforms at $x$ coordinate axis from $(-60,0)$ to $(60,0)$. The results of simulated experiment are shown in TableI.

TABLE I. RESULTS OF SIMULATED EXPERIMENT

\begin{tabular}{|c|c|c|}
\hline & $\begin{array}{c}\text { Pages-Zamora } \\
\text { Algorithm }\end{array}$ & Fuzzy Algorithm \\
\hline $\begin{array}{c}\text { Location } \\
\left(x_{T}, y_{T}\right)\end{array}$ & $(-0.2316,101.3226)$ & $(-0.9257,99.7269)$ \\
\hline error & 1.34272 & 0.965097 \\
\hline Standard & & \\
$\begin{array}{c}\text { Deviation } \\
\left(S_{x_{T}}, S_{y_{T}}\right)\end{array}$ & $(0.6641,2.7812)$ & $(0.790207,1.96773)$ \\
\hline E.E.P & 2.85939 & 2.12047 \\
\hline
\end{tabular}

According to the Table I, we can conclude that the results by using Fuzzy Algorithm are better than the results by using Pages-Zamora Algorithm.

\section{VI SUMMARY}

In this paper, we raise a fuzzy method for the passive location of emitter based on membership function to achieve the location. The simulation experiment demonstrates that the results by using Fuzzy Algorithm are better than the results by using Pages-Zamora Algorithm.

\section{REFERENCES}

[1] ZhongKang Sun, Guo FuCheng, Feng WangDao. The Research on Single Passive Location. Defense Industry Press, Beijing,2008.

[2] Richard A. Poisel. Electronic Warfare Target Location Methods. Publishing House of Electronic Industry, Beijing,2008.

[3] Yang Lunbiao, Gao Yingyi. Principle and Application of Fuzzy Maths[M]. HuaNan Science and Technology University Press, 2005.

[4] LaiZhao Hu. The Research on Passive Location. Defense Industry Press, Beijing,2004.

[5] LuCheng Pei. The Research on Monte Carlo Method. The National Defense Science and Technology University Press, 